\title{
F-box proteins regulate ethylene signaling and more
}

\author{
Xuelu Wang, ${ }^{1}$ Hongzhi Kong, ${ }^{2}$ and Hong $\mathrm{Ma}^{1,3,4,5,6}$ \\ ${ }^{1}$ State Key Laboratory of Genetic Engineering, Institute of Plant Biology, School of Life Sciences, Fudan University, Shanghai \\ 200433, China; ${ }^{2}$ State Key Laboratory of Systematics and Evolution, Institute of Botany, Chinese Academy of Sciences, Beijing \\ 100093, China; ${ }^{3}$ Center for Evolutionary Biology, Fudan University, Shanghai 200433 China; ${ }^{4}$ Institutes of Biomedical Sciences, \\ Fudan University, Shanghai 200032 China; ${ }^{5}$ Department of Biology, the Huck Institutes of the Life Sciences, The Pennsylvania \\ State University, University Park, Pennsylvania 16802, USA
}

Ethylene regulates several aspects of plant development, such as fruit ripening. In this issue of Genes \& Development, Qiao and colleagues (pp. 512-521) report that the stability of the ethylene signaling protein EIN2 is modulated by two F-box proteins ETP1/2, reminiscent of the finding that another regulator of ethylene response, EIN3, is also targeted by the F-box proteins EBF1/2. ETP1/2 and EBF1/2 show distinct phylogenetic patterns, suggesting that they have different evolutionary constraints.

Ethylene is a gaseous molecule that regulates many aspects of plant development and physiology, including seedling development, leaf and flower senescence, fruit ripening, floral sex determination, root hair development, and biotic and abiotic stress responses (Guo and Ecker 2004; Kendrick and Chang 2008; Zhu and Guo 2008). In dark grown Arabidopsis seedlings, ethylene induces a characteristic morphological syndrome called triple response, with a shortened and thickened seedling stem called a hypocotyl and an exaggerated apical hook. This morphology allows the seedlings that develop in the soil and without light exposure to overcome the pressure of soil and emerge to the surface. In addition, many fruits, such as bananas and tomatoes, become softened in the presence of ethylene; this property of fruit ripening has been exploited such that fruits can be harvested and transported prior to maturity and then artificially "ripened" near the retailing locations. In cucumber and other members of the melon family, male and female flowers develop from the same immature flowers with primordia for both male and female reproductive organs, the stamens and pistil. Under the influence of ethylene, bisexual immature cucumber flowers undergo programmed cell death in the stamens, resulting in the formation of

[Keywords: Hormone; protein degradation; signal transduction; plant] ${ }^{6}$ Corresponding author.

E-MAIL hongma@fudan.edu.cn or hxm16@psu.edu; FAX (814) 863-8082. Article is online at http://www.genesdev.org/cgi/doi/10.1101/gad.1781609. female flowers with a functional mature pistil, whereas male flowers form in the absence of ethylene (Trebitsh et al. 1997; Hao et al. 2003).

Genetic studies have identified a number of key components of the ethylene signaling pathway, including membrane-localized ethylene receptors, a Raf-like protein kinase, MAP kinases (MAPKs) and MAPK kinases (MAPKKs), and transcriptional regulators (Fig. 1; Guo and Ecker 2004; Kendrick and Chang 2008; Zhu and Guo 2008). It has been shown that ethylene inactivates ethylene receptors, such as ETR1 and ETR2, which are redundant negative regulators of ethylene responses (Kendrick and Chang 2008). Dominant mutations in these genes prevent receptors from binding to ethylene, allowing the receptors to function even in the presence of ethylene, whereas recessive loss-of-function mutations in a single receptor gene do not cause obvious defects because of functional redundancy. Plants with recessive mutations in multiple receptor genes exhibit a constitutive ethylene response, indicating that the receptors are indeed negative regulators of the ethylene response. Downstream from the ethylene receptors is the Raf homolog CTR1, which also negatively regulates ethylene response. Recessive mutations in CTR 1 cause a constitutive triple response, and are epistatic to the dominant ethylene-insensitive mutations in the receptor genes. Multiple genes are known to encode MAPKs and MAPKKs in Arabidopsis and other plants, and molecular genetic studies support their role in ethylene response, at least in some physiological processes.

Furthermore, early mutant screens uncovered recessive ein2 and ein3 mutants, both are insensitive to ethylene (Chao et al. 1997). Both ein2 and ein3 mutations are epistatic to the ctr1 mutation, suggesting that EIN2 and EIN3 act downstream from CTR1 and are important positive regulators of the ethylene response. EIN3 encodes a transcription factor and has been shown to bind to the primary ethylene response element (ERE) in the promoter region of the ethylene early responsive gene ERF1 (Solano et al. 1998). ERF1 and its paralogs are members of the AP2 family of DNA-binding proteins that were initially identified as proteins that bind to the 


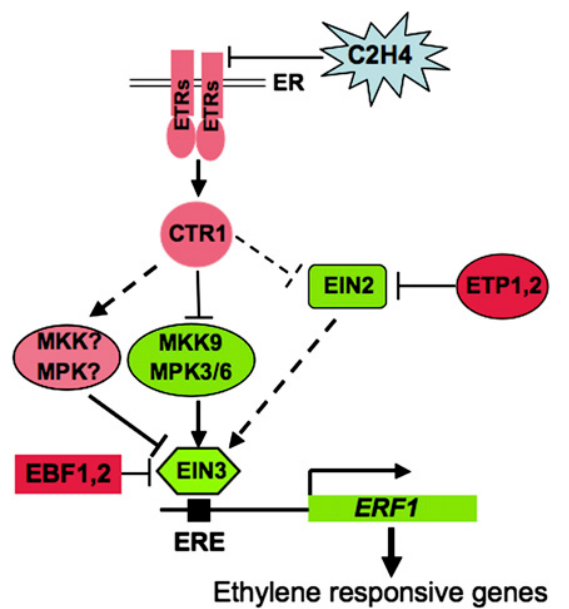

Figure 1. The ethylene signaling pathway and the regulation of its components by F-box proteins. Ethylene is perceived at the endoplasmic reticulum membrane by a family of ethylene receptors (ETRs, including ETR1 and ERS1). In the absence of ethylene, the ethylene receptors activate the RAF-like MAPKK kinase CTR1, which represses ethylene signaling by inhibiting the activity of MKK9-MPK3/6 module and the membrane localized EIN2. In addition, another unknown MAPK module may be activated by CTR 1 and phosphorylate the T592 site of EIN3, leading to the degradation of EIN3 mediated through the two F-box proteins EBF1 and EBF2. Ethylene binding to its receptors inhibits the activity of receptors and CTR1, and leads to the activation of the MKK9-MPK3/6 module, which phosphorylates EIN3 on T174, leading to the stabilization and accumulation of EIN3. Then EIN3 enhances the expression of ERF1 through binding to the ERE element of the promoter region of ERF1, which further regulates the expression of ethylene-responsive genes. In the presence of ethylene, EIN2 is activated and promotes ethylene-induced responses through EIN3 using an unknown mechanism. The stability of EIN2 is negatively regulated by the two newly discovered F-box proteins ETP1 and ETP2.

ERE motifs found in promoters of ethylene-induced genes and activate these genes. Therefore, EIN3 and ERFs represent two levels in a transcriptional network for ethylene response.

The EIN2 gene encodes a membrane protein whose $\mathrm{N}$-terminal region has limited sequence similarity to mammalian metal transporters called NRAMPs (Alonso et al. 1999). The C-terminal region of the EIN2 protein is well conserved in flowering plants and contains $\sim 850$ amino acid residues; this region was found to confer constitutive ethylene response in transgenic Arabidopsis plants (Varma Penmetsa et al. 2008). Although genetic studies clearly indicated that EIN2 is an important component of the ethylene signaling pathway, it has been unclear how ethylene regulates its activity. In this issue of Genes \& Development, Qiao et al. (2009) describe results from a comprehensive study that sheds new light on the regulation of EIN2 and ethylene signaling.

Because ethylene regulates the expression of many genes, including $E R F S$, it was reasonable to hypothesize that ethylene might activate EIN2 transcription. However, Alonso et al. (1999) showed previously that EIN2
mRNA levels were not affected by ethylene, suggesting that transcriptional control was not a major mechanism for regulating EIN2. How, then, is ethylene connected to EIN2 activity? Qiao et al. (2009) show that EIN2 is regulated by ethylene at the level of protein stability; EIN2 was found to be unstable and its stability was enhanced by proteasome inhibitors and by ethylene. The amount of EIN2 protein was increased in the ctr1 mutant, but reduced in the etr1 mutant, suggesting that EIN2 stability is regulated by known components of the ethylene signaling pathway.

How is the stability of the EIN2 protein controlled? To address this question, Qiao et al. (2009) used the yeast two-hybrid method to search for proteins that interact with EIN2 using its conserved C-terminal region. They found an EIN2-interacting protein (ETP1) that contains an F-box domain; in addition, they showed that a paralog of ETP1, ETP2, also can interact with EIN2. A number of F-box proteins are known to be components of protein ubiquitin ligases called SCF complexes and are important for specifying specific substrates. This suggests that ETP1 and ETP2 might facilitate the ubiquitination of EIN2, thereby regulating its degradation by the proteasome. To test this idea, Qiao et al. (2009) used plants that had altered the function of ETP1 and ETP2, and found that EIN2 accumulated to abnormally high levels in plants with reduced ETP1/2 function and was hardly detectable in plants with elevated ETP1/2 function. Moreover, overexpression of ETP1 or ETP2 resulted in a decrease in response to ethylene, whereas reduced ETP1/2 function caused changes in plant morphology and gene expression similar to those induced by ethylene treatment. They also showed that ethylene could induce a reduction of ETP1 and ETP2 protein levels, but had no detectable effect on ETP1/2 mRNA, suggesting that ETP1/2 themselves are regulated post-transcriptionally.

In short, Qiao et al. (2009) provided strong genetic and biochemical evidence that the F-box proteins ETP1/2 are important for modulating ethylene signaling, and that they likely regulate EIN2 protein levels by facilitating its ubiquitination. In fact, EIN2 is not the first key mediator of ethylene signaling that is regulated at the level of protein stability and ETP1/2 are not the first F-box proteins found to be important for this process. Previous studies showed that the level of the EIN3 protein, but not EIN3 mRNA, increased following ethylene treatment, indicating that ethylene can stabilize the EIN3 protein (Guo and Ecker 2003). In addition, inhibition of proteasome leads to the accumulation of EIN3, suggesting that EIN3 is degraded by the proteasome (Guo and Ecker 2003). Using the yeast two hybrid method, two EIN3-binding proteins (EBF1 and EBF2) were identified and found to be F-box proteins (Guo and Ecker 2003; Potuschak et al. 2003); moreover, EBF1 and EBF2 can interact with putative SCF subunits ASKs, suggesting that EBF1 and EBF2 are indeed components of SCF complexes (Guo and Ecker 2003; Potuschak et al. 2003; Gagne et al. 2004). Mutations in EBF1 and EBF2 lead to accumulation of the EIN3 protein and enhanced ethylene responses (Guo and Ecker 2003; Potuschak et al. 2003; Gagne et al. 2004). 
Kinetic analyses of $e b f 1$ and $e b f 2$ mutant response to ethylene indicate that EBF1 primarily degrades EIN3 with no or low ethylene signaling, whereas EBF2 plays a larger role in degrading EIN3 after ethylene responses have been activated (Binder et al. 2007). It is unknown whether ethylene can regulate the activity of $\mathrm{EBF} 1 / 2$, but the transcript level of EBF $1 / 2$ is negatively regulated by EIN5/XRN4, which encodes an exoribonuclease (Olmedo et al. 2006; Potuschak et al. 2006).

More recently, it was reported that the stability of EIN3 appears to be regulated by phosphorylation (Yoo et al. 2008). It was found that EIN3 could be phosphorylated by two MAPKs, MPK3 and MPK6, and MPK3/6 can be activated by MKK9; in addition, phosphorylation can stabilize the EIN3 protein. In contrast, a constitutively active form of CTR1 can cause phosphorylation of EIN3 at another site, leading to EIN3 degradation (Yoo et al. 2008), indicating that the negative regulator CTR1 in the ethylene signaling pathway can promote EIN3 degradation likely through the activation of another unknown MAPK module. Although it is still unclear how these phosphorylation sites alter the stability of EIN3, it is likely that $\mathrm{EBF} 1 / 2$ has differential affinity to the different forms of EIN3. In addition, the ethylene receptor ETR2 might also be degraded by the proteasome (Chen et al. 2007), although the relevant ubiquitin ligase has not yet been identified.

Because ethylene is known to affect development and physiology of many plants, key components of ethylene signaling are likely conserved among divergence plant species (Wilkinson et al. 1995; Varma Penmetsa et al. 2008; Qiao et al. 2009; for review, see Dreher and Callis 2007). For example, both the Arabidopsis and tomato ethylene receptors are members of the same gene family (Wilkinson et al. 1995). EIN2 is also conserved among flowering plants and a putative homolog was identified in an algae (Varma Penmetsa et al. 2008; Qiao et al. 2009). Therefore, SCF-mediated ubiquitination and proteasomecatalyzed degradation of ethylene signaling proteins also might have been conserved mechanisms. SCF subunit F-box proteins are encoded by multigene families; indeed, a single flowering plant species contains several hundred F-box genes. Recently, phylogenetic studies of the F-box genes from Arabidopsis, poplar and rice showed that the F-box genes in flowering plants can be grouped into 42 gene families according to evolutionary relationship and protein domain organization (Xu et al. 2009). In addition, different gene families have vastly different rates of gene duplication. Some of them have a small number of genes that are relatively stable between Arabidopsis, poplar, and rice. Others have drastic changes after the divergence of these three plant lineages, and often have a large number of species-specific genes without clear orthologs in other plants (Xu et al. 2009).

To learn more about the evolution of the ethylene signaling F-box genes, homologs of EBF1/2 and ETP1/2 were identified from poplar, rice, and other selected flowering plants, and were used constructed phylogenetic trees (Fig. 2). The tree for EBF1/2 homologs has a typical topology of relative conservative relationships (Fig. 2A), suggesting that their functions are largely stable during
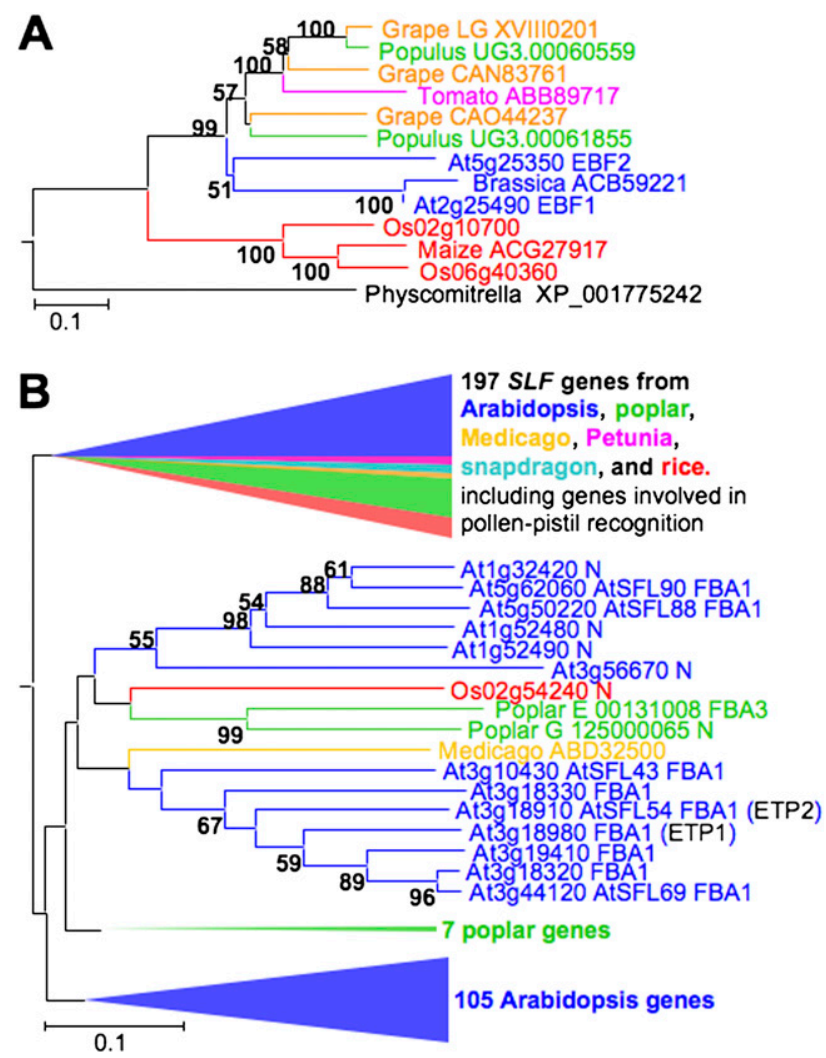

Figure 2. Phylogenetic relationships of F-box genes involved in ethylene signaling and their homologs. The neighbor joining (NJ) trees were built in MEGA 4 on the basis of protein sequences in the F-box domain region. The Arabidopsis EBF1/2 and ETP1/2 genes belong to the LRR_7 and FBA families, respectively, of the F-box superfamily (Xu et al. 2009). Like EBF1/2, members of the LRR_7 family usually encode proteins with one to a few LRR domains C-terminal of the highly conserved F-box domain. In contrast, proteins of ETP1/2 and other FBA family members usually have a FBA domain C-terminal of the F-box domain. The evolutionary history of EBF1/2 is relatively simple, because orthology can be relatively easily inferred for genes from different plants $(A)$. The conservative evolution implies that the EBF1/2 proteins and their close homologs might function to interact with relatively stable substrates for ubiquitination. In the ETP1/2 case, however, it has been difficult to infer orthology because repeated duplications have occurred during plant evolution $(B)$. Several genes of this same clade were annotated as relatives of the $S$-locus F-box genes. Yet, this tree suggests that they are only distantly related to $S$-locus F-box genes and, therefore, might have distinct functions.

flowering plant history. In contrast, genes related to ETP1/ 2 have a more complex pattern of gene duplication (Fig. 2B). ETP1/2 are related to genes (SLFs) that were found to be important for the male component of a pollen-pistil interaction called self-incompatibility in Antirrhinum, petunia, and members of the rose family (McClure and Franklin-Tong 2006). In Arabidopsis, $>100$ genes were found to be related to the SLF genes (Wang et al. 2004; Xu et al. 2009). Further phylogenetic analysis showed that the ETP $1 / 2$ genes are in a clade that is distinct from the clade that contains the SLF genes. In addition, both 
clades include members from Arabidopsis, poplar and rice, suggesting that both clades each originated from an ancestral gene in the most recent common ancestor of these three species. Furthermore, the ETP1/2 clade contains several Arabidopsis genes that are closely related to ETP1/2, suggesting that they might also play a role in regulating EIN2 stability. The topology of the ETP1/2 clade also indicates that there have been recent duplications, suggesting that they have evolved more rapidly than the EBF1/2 genes. At the same time, the putative ETP1/2 homologous F-box proteins in rice and poplar might be involved in targeting the EIN2 homologs in these plants.

In addition to EBF1/2 and ETP1/2, several other F-box proteins also have been found to control the degradation of key components in other hormonal pathways. In the auxin signaling pathway, a small family of F-box proteins, TIR1 (Transport Inhibitor Response 1) and its paralogs, AFBs (Auxin signaling F-Box proteins), facilitates ubiquitination of key transcription repressors, the AUX/ IAA proteins, in an auxin-dependent manner (Dharmasiri et al. 2005a,b; Kepinski and Leyser 2005; Tan et al. 2007), causing their rapid degradation by the proteasome. This releases the inhibitory effect of AUX/IAA on the auxin response factors (ARFs) to promote the expression of auxin-responsive genes (Leyser et al. 1993; Abel et al. 1994; Gray et al. 2001; Guilfoyle and Hagen 2007). Similarly, the receptor COI1 (Coronatine-Insensitive 1) for the plant defense hormone, jasmonic acid (JA) is also an F-box protein (Xie et al. 1998; for review, see Browse 2008). Genetic and biochemical data indicate that jasmonoylisoleucine (JA-Ile), the active form of JA, induces the binding of COIl to the JAZ (Jasmonate ZIM domain) transcriptional repressors, leading to the degradation of the JAZ proteins by the proteasome (Chini et al. 2007; Thines et al. 2007; Katsir et al. 2008). Therefore, both auxin and JA directly bind to F-box proteins and regulate their ability to bind to their substrates, which are repressors of the respective hormone-induced genes.

In the case of the signaling pathway for the hormone gibberellic acid $(\mathrm{GA})$, the regulation is slightly more complex. GA response also involves transcriptional activation, and requires GA-induced degradation of a class of DELLA proteins, which represses GA-activated genes (Schwechheimer 2008), similar to the proteasome-mediated degradation of AUX/IAA and JAZ proteins in the auxin and JA pathways, respectively. The degradation of DELLA proteins is also facilitated by F-box proteins called SLEEPY1 (SLY1) in Arabidopsis (McGinnis et al. 2003; Dill et al. 2004) and GID2 in rice (Sasaki et al. 2003). However, the GA hormone does not bind to the F-box proteins directly. Instead, the GA receptor, GID1 is a novel soluble protein (Ueguchi-Tanaka et al. 2005, 2007). The binding of GA to GID1 dramatically enhances the interaction of the F-box protein GID2/SLY1 with DELLA proteins (Griffiths et al. 2006), providing a mechanism for GA induction of DELLA protein degradation. Thus, acting via the GA receptor GID1, GA activates the function of $\mathrm{SCF}^{\mathrm{SLY} 1 / \mathrm{GID} 2}$ to target DELLA proteins for ubiquitinmediated proteolysis.
The levels of key components of the brassinosteroid signaling pathway, the transcription factors BES1/ BZR1-especially the phosphorylated form-are also regulated by the proteasome (He et al. 2002; Wang et al. 2002; Yin et al. 2002). It was reported recently that a negative regulator Brassinosteroid-Insensitive 2 (BIN2) of the brassinosteroid signaling pathway is also regulated by protein degradation (Peng et al. 2008). However, the specific ubiquitin ligases responsible for the degradation of either BES1/ZBR1 or BIN2 have not been identified. Recently, F-box proteins or other ubiquitin ligases have been found to mediate signaling of other hormones, including abscisic acid (Zhang et al. 2005; Stone et al. 2006) and a recently discovered hormone regulating plant branching (Mouchel and Leyser 2007).

Qiao et al. (2009) report new results that demonstrate an important function for F-box proteins in regulating ethylene responses. This represents the latest chapter in a series of discoveries concerning the crucial roles that F-box proteins and the proteasome play in several plant hormonal signaling pathways. From these studies, it is amply clear that regulated protein degradation is a highly effective mechanism for the regulation of signal transduction pathways. In animals, hormonal signaling and transduction of neural transmitters are often mediated by transmembrane receptors and protein kinases, which catalyze reversible changes of proteins, allowing protein activities to be controlled in a transient fashion. This is probably important for rapid animal reactions that are important in a predator and prey relationship. In contrast, plants cannot move and respond to much slower environmental changes by altering developmental programs. It is possible that protein degradation is an effective and stable means of regulating plant signaling processes, which often cause morphological changes over a period of time.

\section{Acknowledgments}

We thank H. Guo (Peking University) for helpful comments. X.W. was supported by the National Natural Science Foundation of China (30671118 and 30871330) and a grant of Shanghai Pujiang Project (07pj14014), H.K. was supported by the National Natural Science Foundation of China (30530090) and the Chinese Academy of Sciences (KSCX2-YW-R-135), and H.M. was supported by the U.S. Department of Energy (DE-FG0202ER15332) and funds from Rijk Zwaan, Fudan University, and the Pennsylvania State University.

\section{References}

Abel, S., Oeller, P.W., and Theologis, A. 1994. Early auxin induced genes encode short lived nuclear proteins. Proc. Natl. Acad. Sci. 91: 326-330.

Alonso, J.M., Hirayama, T., Roman, G., Nourizadeh, S., and Ecker, J.R. 1999. EIN2, a bifunctional transducer of ethylene and stress responses in Arabidopsis. Science 284: 21482152.

Binder, B., Walker, J., Gagne, J., Emborg, T., Hemmann, G., Bleecker, A., and Vierstra, R. 2007. The Arabidopsis EIN3 binding F-box proteins EBF1 and EBF2 have distinct but overlapping roles in ethylene signaling. Plant Cell 19: 509523. 
Browse, J. 2008. Jasmonate passes muster: A receptor and targets for the defense hormone. Annu. Rev. Plant Biol. doi: 10.1146/ annurev.arplant.043008.092007.

Chao, Q., Rothenberg, M., Solano, R., Roman, G., Terzaghi, W., and Ecker, J.R. 1997. Activation of the ethylene gas response pathway in Arabidopsis by the nuclear protein ETHYLENEINSENSITIVE3 and related proteins. Cell 89: 1133-1144.

Chen, Y., Shakeel, S.N., Bowers, J., Zhao, X.C., Etheridge, N., and Schaller, G.E. 2007. Ligand-induced degradation of the ethylene receptor ETR2 through a proteasome-dependent pathway in Arabidopsis. J. Biol. Chem. 282: 24752-24758.

Chini, A., Fonseca, S., Fernandez, G., Adie, B., Chico, J.M., Lorenzo, O., Garcia-Casado, G., Lopez-Vidriero, I., Lozano, F.M., Ponce, M.R., et al. 2007. The JAZ family of repressors is the missing link in jasmonate signalling. Nature 448: 666671.

Dharmasiri, N., Dharmasiri, S., and Estelle, M. 2005a. The F-box protein TIR 1 is an auxin receptor. Nature 435: 441-445.

Dharmasiri, N., Dharmasiri, S., Weijers, D., Lechner, E., Yamada, M., Hobbie, L., Ehrismann, J.S., Jurgens, G., and Estelle, M. 2005b. Plant development is regulated by a family of auxin receptor F box proteins. Dev. Cell 9: 109-119.

Dill, A., Thomas, S.G., Hu, J., Steber, C.M., and Sun, T.-p. 2004. The Arabidopsis F-box protein SLEEPY1 targets GA signaling repressors for GA-induced degradation. Plant Cell 16: 1392-1405.

Dreher, K. and Callis, J. 2007. Ubiquitin, hormones and biotic stress in plants. Ann. Bot. (Lond.) 99: 787-822.

Gagne, J., Smalle, J., Gingerich, D., Walker, J., Yoo, S., Yanagisawa, S., and Vierstra, R. 2004. Arabidopsis EIN3-binding F-box 1 and 2 form ubiquitin-protein ligases that repress ethylene action and promote growth by directing EIN3 degradation. Proc. Natl. Acad. Sci. 101: 6803-6808.

Gray, W.M., Kepinski, S., Rouse, D., Leyser, O., and Estelle, M. 2001. Auxin regulates SCFTIR1-dependent degradation of AUX/IAA proteins. Nature 414: 271-276.

Griffiths, J., Murase, K., Rieu, I., Zentella, R., Zhang, Z.L., Powers, S.J., Gong, F., Phillips, A.L., Hedden, P., Sun, T.P., et al. 2006. Genetic characterization and functional analysis of the GID1 gibberellin receptors in Arabidopsis. Plant Cell 18: 3399-3414.

Guilfoyle, T.J. and Hagen, G. 2007. Auxin response factors. Curr. Opin. Plant Biol. 10: 453-460.

Guo, H. and Ecker, J.R. 2003. Plant responses to ethylene gas are mediated by SCFEBF1/EBF2-dependent proteolysis of EIN3 transcription factor. Cell 115: 667-677.

Guo, H. and Ecker, J.R. 2004. The ethylene signaling pathway: New insights. Curr. Opin. Plant Biol. 7: 40-49.

Hao, Y.J., Wang, D.H., Peng, Y.B., Bai, S.L., Xu, L.Y., Li, Y.Q., Xu, Z.H., and Bai, S.N. 2003. DNA damage in the early primordial anther is closely correlated with stamen arrest in the female flower of cucumber (Cucumis sativus L.). Planta 217: 888-895.

He, J.X., Gendron, J.M., Yang, Y., Li, J., and Wang, Z.Y. 2002. The GSK3-like kinase BIN2 phosphorylates and destabilizes BZR1, a positive regulator of the brassinosteroid signaling pathway in Arabidopsis. Proc. Natl. Acad. Sci. 99: 1018510190.

Katsir, L., Chung, H.S., Koo, A.J., and Howe, G.A. 2008. Jasmonate signaling: A conserved mechanism of hormone sensing. Curr. Opin. Plant Biol. 11: 428-435.

Kendrick, M.D. and Chang, C. 2008. Ethylene signaling: New levels of complexity and regulation. Curr. Opin. Plant Biol. 11: 479-485.

Kepinski, S. and Leyser, O. 2005. The Arabidopsis F-box protein TIR1 is an auxin receptor. Nature 435: 446-451.
Leyser, H.M.O., Lincoln, C.A., Timpte, C., Lammer, D., Turner, J., and Estelle, M. 1993. Arabidopsis auxin resistance gene AXR1 encodes a protein related to ubiquitin activating enzyme E1. Nature 364: 161-164.

McClure, B.A. and Franklin-Tong, V. 2006. Gametophytic selfincompatibility: Understanding the cellular mechanisms involved in 'self' pollen tube inhibition. Planta 224: 233-245.

McGinnis, K.M., Thomas, S.G., Soule, F.D., Strader, L.C., Zale, J.M., Sun, T.-p., and Steber, C.M. 2003. The Arabidopsis SLEEPY1 gene encodes a putative F-box subunit of an SCF E3 ubiquitin ligase. Plant Cell 15: 1120-1130.

Mouchel, C.F. and Leyser, O. 2007. Novel phytohormones involved in long-range signaling. Curr. Opin. Plant Biol. 10: 473-476.

Olmedo, G., Guo, H., Gregory, B., Nourizadeh, S., AguilarHenonin, L., Li, H., An, F., Guzman, P., and Ecker, J. 2006. ETHYLENE-INSENSITIVE5 encodes a $5^{\prime} \rightarrow 3^{\prime}$ exoribonuclease required for regulation of the EIN3-targeting F-box proteins EBF1/2. Proc. Nat1. Acad. Sci. 103: 13286-13293.

Peng, P., Yan, Z., Zhu, Y., and Li, J. 2008. Regulation of the Arabidopsis GSK3-like kinase Brassinosteroid-insensitive 2 through proteasome-mediated protein degradation. Mol. Plant 1: 338-346.

Potuschak, T., Lechner, E., Parmentier, Y., Yanagisawa, S., Grava, S., Koncz, C., and Genschik, P. 2003. EIN3-dependent regulation of plant ethylene hormone signaling by two Arabidopsis F Box proteins: EBF1 and EBF2. Cell 115: 679-689.

Potuschak, T., Vansiri, A., Binder, B., Lechner, E., Vierstra, R., and Genschik, P. 2006. The exoribonuclease XRN4 is a component of the ethylene response pathway in Arabidopsis. Plant Cell 18: 3047-3057.

Qiao, H., Chang, K.N., Yazaki, J., and Ecker, J.R. 2009. Interplay between ethylene, ETP1/ETP2 F-box proteins, and degradation of EIN2 triggers ethylene responses in Arabidopsis. Genes \& Dev. (this issue). doi: 10.1101/gad.1765709.

Sasaki, A., Itoh, H., Gomi, K., Ueguchi-Tanaka, M., Ishiyama, K., Kobayashi, M., Jeong, D.-H., An, G., Kitano, J., Ashikari, M., et al. 2003. Accumulation of phosphorylated repressor for gibberellin signaling in an F-box mutant. Science 299: 1896 1898.

Schwechheimer, C. 2008. Understanding gibberellic acid signaling-Are we there yet? Curr. Opin. Plant Biol. 11: 9-15.

Solano, R., Stepanova, A., Chao, Q., and Ecker, J.R. 1998. Nuclear events in ethylene signaling: A transcriptional cascade mediated by ETHYLENE-INSENSITIVE3 and ETHYLENERESPONSE-FACTOR1. Genes \& Dev. 12: 3703-3714.

Stone, S.L., Williams, L.A., Farmer, L.M., Vierstra, R.D., and Callis, J. 2006. KEEP ON GOING, a RING E3 ligase essential for Arabidopsis growth and development, is involved in abscisic acid signaling. Plant Cell 18: 3415-3428.

Tan, X., Calderon-Villalobos, L.I.A., Sharon, M., Zheng, C.X., Robinson, C.V., Estelle, M., and Zheng, N. 2007. Mechanism of auxin perception by the TIR1 ubiquitin ligase. Nature 446: 640-645.

Thines, B., Katsir, L., Melotto, M., Niu, Y., Mandaokar, A., Liu, G.H., Nomura, K., He, S.Y., Howe, G.A., and Browse, J. 2007. JAZ repressor proteins are targets of the SCFCOI1 complex during jasmonate signalling. Nature 448: 661-665.

Trebitsh, T., Staub, J.E., and O'Neill, S.D. 1997. Identification of a 1-aminocyclopropane-1-carboxylic acid synthase gene linked to the female (F) locus that enhances female sex expression in cucumber. Plant Physiol. 113: 987-995.

Ueguchi-Tanaka, M., Ashikari, M., Nakajima, M., Itoh, H., Katoh, E., Kobayashi, M., Chow, T.Y., Hsing, Y.I., Kitano, H., Yamaguchi, I., et al. 2005. GIBBERELLIN INSENSITIVE $D W A R F 1$ encodes a soluble receptor for gibberellin. Nature 437: 693-698. 
Ueguchi-Tanaka, M., Nakajima, M., Katoh, E., Ohmiya, H., Asano, K., Saji, S., Hongyu, X., Ashikari, M., Kitano, H., Yamaguchi, I., et al. 2007. Molecular interactions of a soluble gibberellin receptor, GID1, with a rice DELLA protein, SLR1, and gibberellin. Plant Cell 19: 2140-2155.

Varma Penmetsa, R., Uribe, P., Anderson, J., Lichtenzveig, J., Gish, J.C., Nam, Y.W., Engstrom, E., Xu, K., Sckisel, G., Pereira, M., et al. 2008. The Medicago truncatula ortholog of Arabidopsis EIN2, sickle, is a negative regulator of symbiotic and pathogenic microbial associations. Plant J. 55: 580-595.

Wang, Z.Y., Nakano, T., Gendron, J., He, J., Chen, M., Vafeados, D., Yang, Y., Fujioka, S., Yoshida, S., Asami, T., et al. 2002. Nuclear-localized BZR1 mediates brassinosteroid-induced growth and feedback suppression of brassinosteroid biosynthesis. Dev. Cell 2: 505-513.

Wang, L., Dong, L., Zhang, Y., Zhang, Y., Wu, W., Deng, X., and Xue, Y. 2004. Genome-wide analysis of $S$-Locus F-boxlike genes in Arabidopsis thaliana. Plant Mol. Biol. 56: 929-945.

Wilkinson, J.Q., Lanahan, M.B., Yen, H.C., Giovannoni, J.J., and Klee, H.J. 1995. An ethylene-inducible component of signal transduction encoded by never-ripe. Science 270: 1807-1809.

Xie, D.X., Feys, B.F., James, S., Nieto-Rostro, M., and Turner, J.G. 1998. COI1: An Arabidopsis gene required for jasmonateregulated defense and fertility. Science 280: 1091-1094.

Xu, G., Ma, H., Nei, M., and Kong, H. 2009. Evolution of F-box genes in plants: Different modes of sequence divergence and their relationships with functional diversification. Proc. Natl. Acad. Sci. 106: 835-840.

Yin, Y.H., Wang, Z.Y., Mora-Garcia, S., Li, J.M., Yoshida, S., Asami, T., and Chory, J. 2002. BES1 accumulates in the nucleus in response to brassinosteroids to regulate gene expression and promote stem elongation. Cell 109: 181-191.

Yoo, S., Cho, Y., Tena, G., Xiong, Y., and Sheen, J. 2008. Dual control of nuclear EIN3 by bifurcate MAPK cascades in C2H4 signaling. Nature 451: 789-795.

Zhang, X., Garreton, V., and Chua, N.H. 2005. The AIP2 E3 ligase acts as a novel negative regulator of ABA signaling by promoting ABI3 degradation. Genes \& Dev. 19: 1535-1543.

Zhu, Z. and Guo, H. 2008. Genetic basis of ethylene perception and signal transduction in Arabidopsis. J. Integr. Plant Biol. 50: $808-815$. 


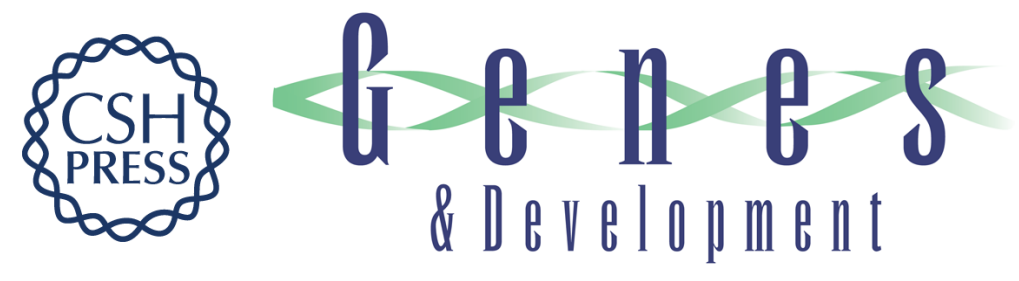

\section{F-box proteins regulate ethylene signaling and more}

\section{Xuelu Wang, Hongzhi Kong and Hong Ma}

Genes Dev. 2009, 23:

Access the most recent version at doi:10.1101/gad.1781609

\section{Related Content Interplay between ethylene, ETP1/ETP2 F-box proteins, and degradation of EIN2 triggers ethylene responses in Arabidopsis \\ Hong Qiao, Katherine N. Chang, Junshi Yazaki, et al. \\ Genes Dev. February , 2009 23: 512-521}

\section{References This article cites 49 articles, 20 of which can be accessed free at:} http://genesdev.cshlp.org/content/23/4/391.full.html\#ref-list-1

Articles cited in:

http://genesdev.cshlp.org/content/23/4/391.full.htmI\#related-urls

\section{License}

Email Alerting

Service

Receive free email alerts when new articles cite this article - sign up in the box at the top right corner of the article or click here.

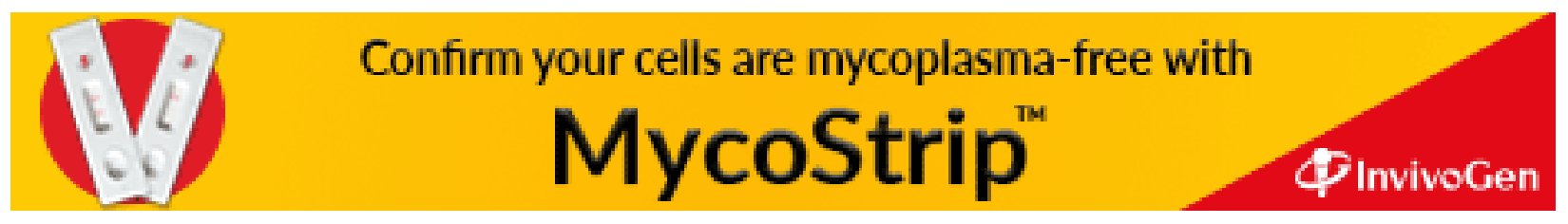

